\title{
Erratum to: Journal of Clinical Monitoring and Computing 2016 end of year summary: cardiovascular and hemodynamic monitoring
}

\author{
Bernd Saugel $^{1} \cdot$ Karim Bendjelid $^{2} \cdot$ Lester A. Critchley $^{3} \cdot$ Steffen Rex $^{4}$. \\ Thomas W. L. Scheeren ${ }^{5}$
}

Published online: 25 January 2017

(C) Springer Science+Business Media Dordrecht 2017

\section{Erratum to: J Clin Monit Comput DOI 10.1007/s10877-017-9976-3}

In the original publication of the article, in Sect. 2.2, the sentence that reads as "TPTD is a well-validated ... femoral vein)" was incorrectly published. The correct sentence should read "TPTD is a well-validated ... femoral artery)".
In Sect. 4, fourth paragraph, the sentence that reads as, "Furthermore, the pleth variability ... index (PI)-values, was recorded" should read as "Furthermore, the pleth variability ... index (PI)-values divided by the maximal PI was recorded".

The online version of the original article can be found under doi:10.1007/s10877-017-9976-3.

\section{Bernd Saugel \\ bernd.saugel@gmx.de; b.saugel@uke.de \\ 1 Department of Anesthesiology, Center of Anesthesiology and Intensive Care Medicine, University Medical Center Hamburg-Eppendorf, Martinistrasse 52, 20246 Hamburg, Germany}

2 Department of Anesthesiology and Intensive Care, Geneva University Hospitals, Geneva, Switzerland

3 Department of Anesthesia and Intensive Care, Prince of Wales Hospital, The Chinese University of Hong Kong, Shatin, Hong Kong

4 Department of Anesthesiology and Department of Cardiovascular Sciences, University Hospitals Leuven, Herestraat 49, 3000 Leuven, Belgium

5 Department of Anesthesiology, University Medical Center Groningen, University of Groningen, Groningen, The Netherlands 